\title{
Stationary rings of vortices in nonrotating Bose-Einstein condensates
}

\author{
D. M. Jezek and P. Capuzzi \\ Departamento de Física, Facultad de Ciencias Exactas y Naturales, Universidad de Buenos Aires, RA-1428 Buenos Aires, Argentina \\ and Consejo Nacional de Investigaciones Científicas y Técnicas, RA-1428 Buenos Aires, Argentina \\ M. Guilleumas and R. Mayol \\ Departament d'Estructura i Constituents de la Matèria, Facultat de Física, Universitat de Barcelona, E-08028 Barcelona, Spain
} (Received 28 May 2008; revised manuscript received 3 October 2008; published 11 November 2008)

\begin{abstract}
We numerically obtain stationary rings of vortices in pancake-shaped Bose-Einstein condensates confined in a three-dimensional nonrotating trap. For this purpose we use a static axisymmetric trapping potential that can sustain locally stable off-axis vortices. We analyze the maximum number of vortices the system can host as a function of the number of particles. We also show that this system provides a very suitable scenario for predicting vortex dynamics in inhomogeneous media. Specifically, on the one hand, we derive an exact and simple analytical expression for the velocity field in a particular vortex position due to the presence of the other vortices of the array. On the other hand, using the fact that in stationary conditions this field should balance all other contributions to the velocity, we investigate the applicability of approximated expressions for the precession velocity obtained in previous works for condensates with a single vortex.
\end{abstract}

DOI: 10.1103/PhysRevA.78.053616

PACS number(s): 03.75.Lm, 03.75.Hh, 03.75.Kk

\section{INTRODUCTION}

The phenomenon of superfluidity is one of the most remarkable features of macroscopic quantum systems where quantized vortices arise as striking topological objects [1]. In trapped Bose-Einstein condensates (BECs), an enormous amount of work, both theoretical and experimental, has been devoted to study the structure, stability, and dynamics of vortices. (For a review of these issues, see, for example, [2-5].)

Since the first production of a single vortex in a confined BEC [6] many fascinating experiments have been performed. Among the most recent works in nonrotating traps, we want to quote those by Scherer et al. [7], and Ryu et al. [8]. In Ref. [7] the authors have reported the observation of vortex formation by merging and interfering multiple BECs produced by a potential well partitioned by a barrier into three sections. Whereas in Ref. [8] a stable persistent flow in a toroidal trap has been observed. In previous experiments, vortex rings have been obtained as a result of the decay of solitons [9], and vortex lines have been generated from the application of topological phase imprinting methods [10]. While a large variety of vortex configurations, from single vortex to vortex lattices, are generated using rotating traps [11-13]. Finally, in recent experiments, more sophisticated anharmonic trapping potentials, as for example harmonic-plusquartic radial confinements have been constructed in order to obtain vortex lattices in fast rotating condensates [12,13]. There, the quartic term in the potential has been introduced to stabilize the system when the angular velocity exceeds the radial angular frequency of the harmonic trap.

From a theoretical point of view some work has been devoted to study different vortex arrays in these harmonicplus-quartic potentials, Refs. [14-18]. In particular in Ref. [14] they have considered a two-dimensional (2D) annularshaped condensate confined by a Mexican hat potential. They have analyzed the energy of a multiply quantized vortex at the center of the trap and they have further studied the appearance of rings of vortices with increasing external ro- tation. The case for a three-dimensional (3D) cigar-shaped BEC has been discussed in [15], whereas in Refs. [16-18] similar vortex arrays have been considered in fast-rotating traps.

On the other hand, stationary vortex-state configurations in harmonic nonrotating traps have been numerically generated using several techniques [19-22]. As in this type of confining potentials there exists no energy barrier between the vortex and the ground state, the arrays of vortices may be stationary but energetically unstable. Moreover, most of the configurations consist of rings of vortex-antivortex pairs and thus in the presence of dissipation these pairs tend to annihilate each other.

To the best of our knowledge no numerical calculation of stationary rings of vortices with the same circulation sense, in nonrotating traps, has been reported so far. In this work we obtain different annular arrays of vortices using a suitable polynomial trapping potential that can sustain metastable offaxis vortices [23] and a numerical phase imprinting method for obtaining such states. An extra goal of this work is to numerically test the available formulas for the precession velocity of vortices without performing temporal evolutions. Recently, analytical works have been devoted to study the dynamics of vortices in spatially inhomogeneous twodimensional superfluids [24-29]. In this paper we consider pancake-shaped condensates and thus the analytical results of these previous studies are expected to be valid. Moreover, we show that the condensate confined in this particular trap provides a very suitable scenario for testing these approximated analytical expressions. To this aim, we calculate the velocity field due to other vortices in a particular vortex position. Since the vortex array we calculate is stationary, this velocity should be equal in modulus to the vortex precession velocity.

This work is organized as follows. In Sec. II we present the trapping potential and the system. In Sec. III we describe the method for numerically producing a ring of vortices. In Sec. IV we derive an analytical expression for the velocity 
field in a particular vortex position due to the presence of the other vortices. In Sec. V we investigate the applicability of the formulas obtained in previous works to describe the velocity field due to the inhomogeneity for more specific systems. Finally, a summary and concluding remarks are offered in Sec. 6.

\section{TRAPPING POTENTIAL}

We consider a Bose-Einstein condensate of atoms confined in $3 \mathrm{D}$ by an external trap $V_{\text {trap }}$. The Gross-Pitaevskii (GP) energy density functional for the system at zero temperature has the standard form

$$
E[\psi]=\int\left(\frac{\hbar^{2}}{2 m}|\nabla \psi|^{2}+V_{\text {trap }}|\psi|^{2}+\frac{1}{2} g|\psi|^{4}\right) d^{3} r,
$$

where $\psi$ is the condensate wave function and $m$ is the atom mass. The coupling constant $g$ is written in terms of the $s$-wave scattering length $a$ as $g=4 \pi a \hbar^{2} / m$.

Variation of $E$ with respect to $\psi^{*}$ keeping the number of particles fixed yields the GP equation

$$
\left(-\frac{\hbar^{2} \nabla^{2}}{2 m}+V_{\text {trap }}+g|\psi|^{2}\right) \psi=\mu \psi
$$

where $\mu$ is the chemical potential.

We use a recently proposed polynomial trapping potential [23], which written in cylindrical coordinates reads as

$$
V_{\text {trap }}(r, z)=\frac{1}{2} m\left(\omega_{r}^{2} \frac{r^{2}\left(r-r_{1}\right)\left(r-r_{2}\right)}{r_{1} r_{2}}+\omega_{z}^{2} z^{2}\right),
$$

where $\omega_{r}$ and $\omega_{z}$ are radial and axial angular frequencies, respectively. As a function of $r$ this trapping potential has two zeroes at $r_{1}$ and $r_{2}$, a local maximum at $R_{-}<r_{1}$, and two local minima at $r=0$ and $R_{+}$with $r_{1}<R_{+}<r_{2}$. We fix the parameters of the trapping potential to $\left(r_{1}, r_{2}\right)=(10,18)$, in units of $a_{\mathrm{ho}}=\sqrt{\hbar /\left(m \omega_{r}\right)}$. Hereafter, we shall always use $a_{\mathrm{ho}}$ as the unit of length. For this set of parameters the extrema are located at $R_{-}=6$ and $R_{+}=15$. We consider a condensate of ${ }^{87} \mathrm{Rb}$ atoms confined in a pancake-shaped trap with $\omega_{r} /(2 \pi)=100 \mathrm{~Hz}$ and $\omega_{z} /(2 \pi)=520 \mathrm{~Hz}$. The $s$-wave scattering length is $a=98.98 a_{0}$, with $a_{0}$ the Bohr radius. Figure 1 shows the ground-state densities $\rho_{0}=\left|\psi_{0}\right|^{2}$ for $z=0$ and $y=0$, as a function of $x$, obtained by solving the GP equation for two different number of atoms, namely $N_{1}=2.5 \times 10^{5}$ and $N_{2}=10^{6}$. Both particle numbers are large enough to assume that the condensate in the radial direction is in the ThomasFermi (TF) regime. Then, the kinetic term can be neglected in Eq. (2) and the ground-state density can be approximated by

$$
\left|\psi_{0}(r, 0)\right|^{2}=\frac{1}{g}\left[\mu-V_{\text {trap }}(r, 0)\right] \Theta\left(\mu-V_{\text {trap }}\right),
$$

where $\Theta$ is the Heaviside function.

From Fig. 1 it may be seen that, in agreement with the TF approximation, the local potential maximum $R_{-}$generates a local minimum in the density. In the above-mentioned work [23] we have shown that the energy of a single vortex as a

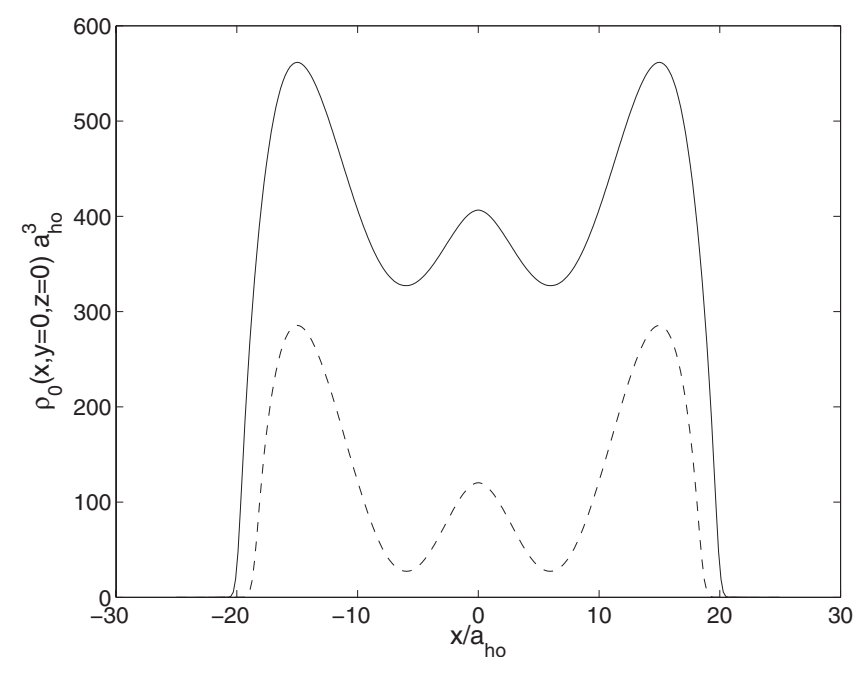

FIG. 1. Ground-state density profile $\rho_{0}$ for $y=0$ and $z=0$ as a function of $x$ for $\left(r_{1}, r_{2}\right)=(10,18)$. The number of particles are $N_{1}$ $=2.5 \times 10^{5}$ (dashed line) and $N_{2}=10^{6}$ (solid line).

function of its position, is approximately proportional to the density of the ground state. Therefore, the local minimum in the density generates a minimum in the vortex energy, too. Thus, a metastable off-axis vortex can be hosted at this position. Whereas, the minimum of the potential at $R_{+}$gives rise to a maximum in the ground-state density and this maximum generates a vortex-energy barrier [23], which in the presence of dissipation will prevent the vortex to spiral away from the condensate.

\section{NUMERICAL GENERATION OF VORTICES}

The method we use to numerically generate the ring of $N_{v}$ vortices is a generalization of the phase imprinting method described in Ref. [23]. In that work we have explained the steps needed to obtain a metastable single-vortex configuration, while in this section several metastable vortices are generated. The procedure consists in minimizing the energy (1) starting from a convenient guess for the vortex state wave function.

We propose two different Ansätze for the initial wave function. One, is to construct the initial $N_{v}$-vortex state as

$$
\psi^{\prime}(\mathbf{r})=\left(\prod_{k=0}^{N_{v}-1} \frac{\left(x-x_{k}\right)+i\left(y-y_{k}\right)}{\sqrt{\left(x-x_{k}\right)^{2}+\left(y-y_{k}\right)^{2}}}\right) \psi_{0}(\mathbf{r}),
$$

with $x_{k}=R_{0} \cos \left(2 \pi k / N_{v}\right)$ and $y_{k}=R_{0} \sin \left(2 \pi k / N_{v}\right)$ where $R_{0}$ $<R_{+}$and $k=0, N_{v}-1$. This wave function can be rewritten as $\psi^{\prime}=e^{i \Sigma_{k} \varphi_{k}} \psi_{0}$, where $\varphi_{k}$ is the azimuthal angle around the axis $\left(x_{k}, y_{k}, z\right)$, representing the vorticity line of the $k$ vortex. Hence, this operation represents phase impressions to the original nonvortex state $\psi_{0}$ around each vorticity line. In summary $\psi^{\prime}$ has the same density profile as $\psi_{0}$ but it carries a velocity field $\mathbf{v}=\Sigma_{k}(\hbar / m) \nabla \varphi_{k}$ which is irrotational everywhere except at the vorticity lines $\left(x_{k}, y_{k}, z\right)$. Using $\psi^{\prime}$ as the initial guess to start the minimization procedure, after the iteration process the system converges to the vortex state solution. It is worth stressing that the converged solution is a 

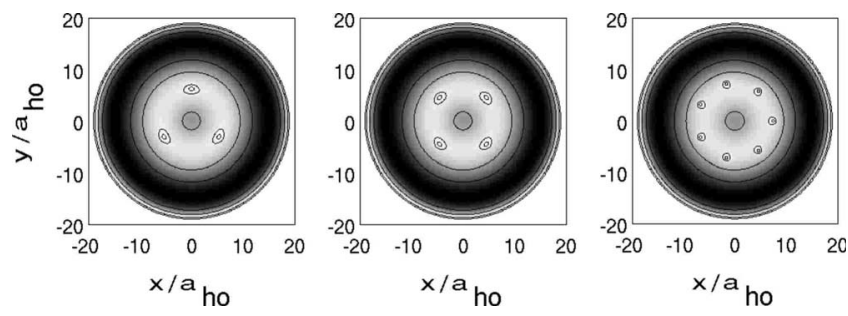

FIG. 2. Equidensity lines at the $(x, y, 0)$ plane, for $N_{v}=3,4$, and 7 vortex state configurations in a condensate with $N_{1}=2.5 \times 10^{5}$ particles.

vortex array with the same number of vortices as the initial Ansatz (5), i.e., $N_{v}$ vortices, but located around a circle with different radius $r_{0}\left(N_{v}\right)$ which increases with the number of vortices imprinted.

The other useful form for imprinting phases in the initial Ansatz is

$$
\psi^{\prime}(\mathbf{r})= \begin{cases}\left(\frac{x+i y}{\sqrt{x^{2}+y^{2}}}\right)^{N_{v}} \psi_{0}(\mathbf{r}) & \text { if } \sqrt{x^{2}+y^{2}}>R_{0}, \\ \psi_{0}(\mathbf{r}) & \text { otherwise, }\end{cases}
$$

with $R_{0}<R_{+}$. In this case $\psi^{\prime}$ has also the same density profile as $\psi_{0}$ but it carries a global velocity field, around the $z$ axis for $r>R_{0}, \mathbf{v}=\hbar / m \nabla \varphi$ which is irrotational everywhere except at the cylinder $x^{2}+y^{2}=R_{0}^{2}$, where $\varphi$ is the azimuthal angle around the $z$ axis.

We have performed several numerical calculations using both methods and we have checked, as expected, that the final state is independent of the initial guess. Moreover, we have included in our calculations more random initial distributions and we found that, for a given final number of vortices the converged state is not modified. For the minimization procedure we have employed the conjugate gradient technique [30].

In Fig. 2 we display equidensity contours for a system with $N_{1}=2.5 \times 10^{5}$ atoms hosting $N_{v}=3,4$, and 7 vortices. While in Fig. 3 (Fig. 4) we show the density profile $\rho_{v}$ as a function of $x$ at $y=z=0$ for a vortex state configuration hosting $N_{v}=7 \quad\left(N_{v}=5\right)$ vortices in a condensate with $N_{1}=2.5$ $\times 10^{5}\left(N_{2}=10^{6}\right)$ atoms. For comparison we show also the corresponding ground-state density $\rho_{0}(x, y=0, z=0)$ as a dashed line. In order to stress the main differences between the density of the $N_{v}$-vortex state and that of the ground state, we depict in the inset of Figs. 3 and 4 the form factor $f=\rho_{v} / \rho_{0}$ around a vortex line. Note that $f$ is almost unity outside the vortex core, thus the density with and without vortices are almost the same except in a narrow region around the cores of the vortices.

Due to both the mutual repulsion between vortices, and the finite size of the energy barrier between the vortex and the ground state [23], there exists an upper limit to the maximum number of vortices, $N_{c}$, that can be sustained by the system. We have calculated the stationary ring configurations by solving the GP for increasing number of vortices from $N_{v}=1$ to $N_{v}=N_{c}$ for several values of $N$. In Fig. 5 we plot the critical number $N_{c}$ as a function of the particle number. As it may be seen $N_{c}$ increases for decreasing number of particles.

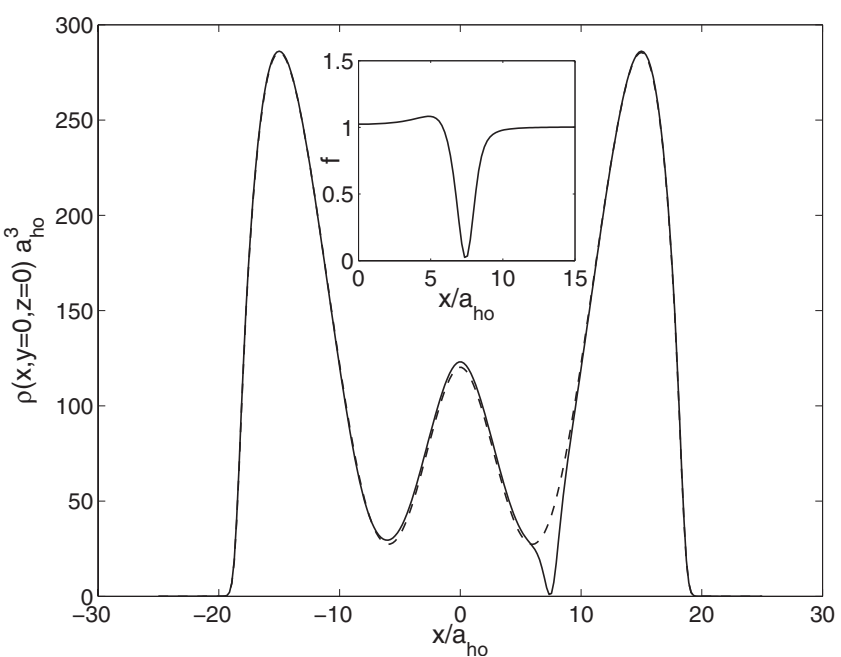

FIG. 3. Density profile as a function of $x$ for $y=0$ and $z=0$ corresponding to the $N_{v}=7$ vortex state configuration depicted in Fig. 2. We also show the ground-state density as a dashed line and the form factor in the inset.

For a number of particles around $N_{0}=2 \times 10^{5}$ the density vanishes at the density minimum and thus the position of the vortices is around this radius independently of their number. In what follows we will focus on particle numbers larger than $N_{0}$ in which case the rings of vortices form in a nonzero ground-state density region, and thus the core of each vortex can be easily observed. In particular, in Table I we show the respective equilibrium radii for $N_{1}$ and $N_{2}$.

\section{VELOCITY FIELD GENERATED BY THE VORTICES}

In this section we derive an expression for the velocity field in the position of a given vortex due to the rest $N_{v}-1$ vortices. As we are working with a pancake-shaped condensate we can assume that the vorticity lines are in the $z$ direc-

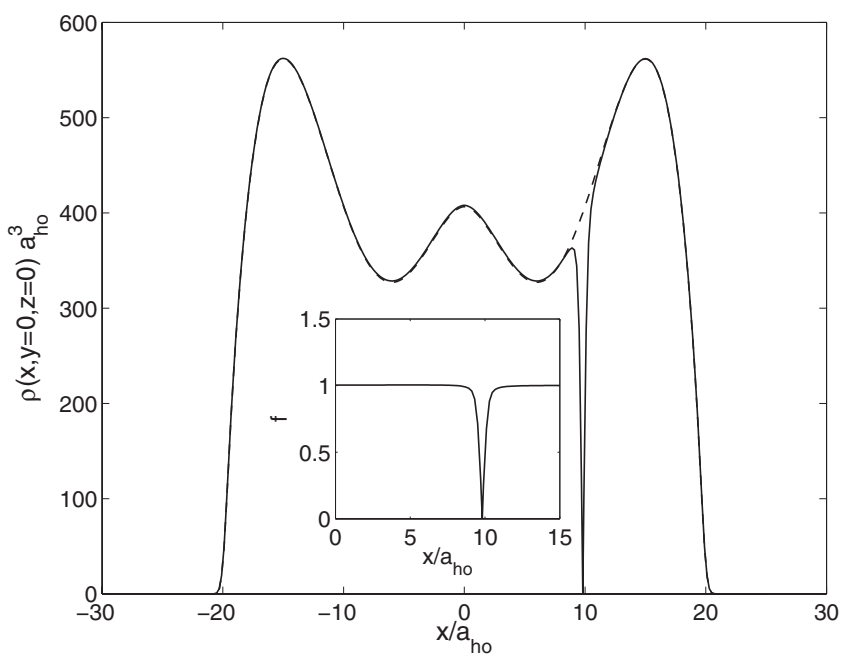

FIG. 4. Density profile as a function of $x$ for $y=0$ and $z=0$ corresponding to a vortex state configuration with $N_{v}=5$ in a condensate with $N_{2}=10^{6}$ atoms. We also depict the ground-state density as a dashed line and the form factor in the inset. 


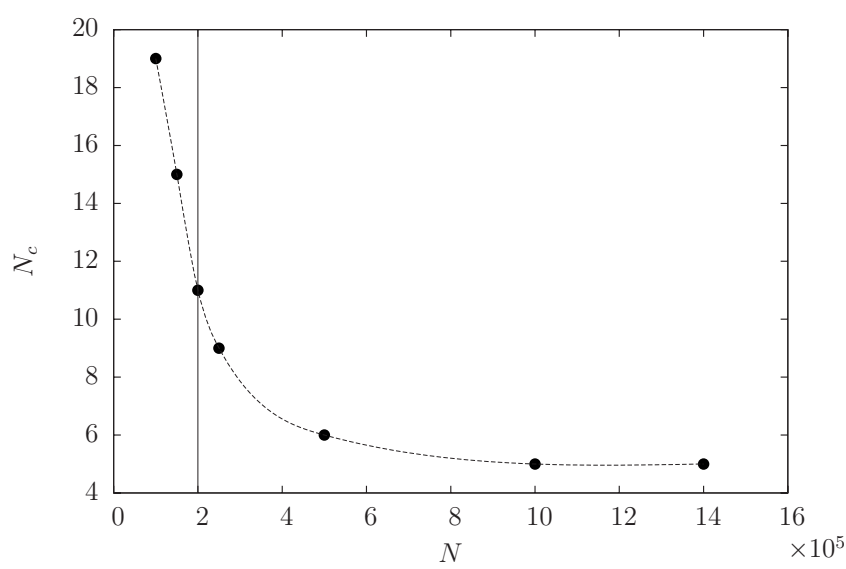

FIG. 5. Critical number of vortices $\left(N_{c}\right)$ as a function of the number of particles $(N)$. The vertical line indicates the value of $N$ below which the ground-state density minimum around $R_{-}$ vanishes.

tion. We will describe the position of the vortex cores in the $x y$ plane $\left(x_{k}, y_{k}\right)$ by using the complex variable

$$
\zeta=x+i y,
$$

and assuming that the vortices are distributed uniformly along a circle of radius $\zeta_{0}$, the position of a $k$ vortex is

$$
\zeta_{k}=x_{k}+i y_{k}=\zeta_{0} e^{i\left(2 \pi k \phi / N_{v}\right)}
$$

where $k=0, \cdots, N_{v}-1$. The complex velocity field $v=v_{x}$ $+i v_{y}$ due to a single vortex $k$ is given by

$$
v_{k}(\zeta)=\frac{\hbar}{m}\left(\frac{-\left(y-y_{k}\right)+i\left(x-x_{k}\right)}{\left(x-x_{k}\right)^{2}+\left(y-y_{k}\right)^{2}}\right) \text {. }
$$

Without loss of generality we may choose the position of the $k=0$ vortex for calculating the velocity field due to the rest of the vortices. Thus its position, $x_{0}=\zeta_{0}, y_{0}=0$, is at the real axis. In this point the contribution of the rest of the vortices is

$$
v\left(\zeta_{0}\right)=\frac{\hbar}{m} \sum_{k=1}^{N_{v}-1} \frac{y_{k}+i\left(\zeta_{0}-x_{k}\right)}{\left(\zeta_{0}-x_{k}\right)^{2}+\left(y_{k}\right)^{2}} .
$$

Using the fact that $\zeta_{k}=-\left(\zeta_{N_{v}-k}\right)^{*}$ it is possible to add together the contributions from $k$ and $N_{v}-k$ vortices yielding the following expression:

$$
v\left(\zeta_{0}\right)=\frac{\hbar}{m} \sum_{k=1}^{\left(N_{v}-1\right) / 2} \frac{2 i\left(\zeta_{0}-x_{k}\right)}{\left(\zeta_{0}-x_{k}\right)^{2}+\left(y_{k}\right)^{2}} .
$$

Then, since the vortices are located at a circle

TABLE I. Equilibrium radii of the rings of vortices obtained for the two number of particles we have considered: $N_{1}=2.5 \times 10^{5}$ and $N_{2}=10^{6}$.

\begin{tabular}{cccccccccc}
\hline \hline$N_{v}$ & 1 & 2 & 3 & 4 & 5 & 6 & 7 & 8 & 9 \\
\hline$N_{1}$ & 6 & 6.05 & 6.18 & 6.41 & 6.67 & 7.05 & 7.33 & 7.86 & 8.72 \\
$N_{2}$ & 6 & 6.81 & 7.69 & 8.63 & 9.80 & & & & \\
\hline
\end{tabular}

$$
x_{k}^{2}+y_{k}^{2}=\zeta_{0}^{2},
$$

the denominator in (11) may be rewritten as

$$
\left(\zeta_{0}-x_{k}\right)^{2}+\left(y_{k}\right)^{2}=2 \zeta_{0}\left(\zeta_{0}-x_{k}\right)
$$

which replaced in Eq. (11) gives

$$
v\left(\zeta_{0}\right)=\frac{\hbar}{m} \sum_{k=1}^{\left(N_{v}-1\right) / 2} \frac{i}{\zeta_{0}}
$$

and thus we finally get the velocity field that feels the $k=0$ vortex due to the presence of the other $N_{v}-1$ vortices, that reads as

$$
v\left(\zeta_{0}\right)=\frac{\hbar}{m} \frac{i\left(N_{v}-1\right) / 2}{\zeta_{0}} .
$$

If the total number of vortices $N_{v}$ is even, the sum in Eq. (11) must be done up to $\left(N_{v}-2\right) / 2$, and the contribution of the unpaired vortex placed at $\zeta=-\zeta_{0}$ must be added separately. This gives

$$
v\left(\zeta_{0}\right)=\frac{\hbar}{m}\left(\frac{i\left(N_{v}-2\right) / 2}{\zeta_{0}}+\frac{i}{2 \zeta_{0}}\right) .
$$

Finally, it follows, that the formula, Eq. (15), is valid for every value of $N_{v}$.

In stationary conditions the net velocity of the vortices is zero and thus the single vortex precession velocity should cancel the drag velocity due to the presence of the other vortices given by Eq. (15). For example, let us analyze the case depicted in Fig. 3 which corresponds to $N_{v}=7$. In the position of the vortex placed at $y_{0}=0$ and $x_{0}=7.33$ the velocity field due to the other vortices using Eq. (15) is $\mathbf{v}$ $=(0.41 \hbar / \mathrm{m}) \hat{\mathbf{y}}$ and as the vortex is at rest this velocity should be balanced by the single-vortex precession velocity. Thus, in general, having numerically computed the equilibrium position $x_{0}$ for an arbitrary number of vortices the above formula provides an indirect method for obtaining the precession velocity of a single vortex at $\mathbf{r}_{0}$. In the following section we treat this issue in more detail.

We note that in the derivation of the velocity field due to the rest of vortices we have not considered the contribution of image vortices. Kim and Fetter [31] have argued that for TF condensates as the density vanishes at the boundary, the particle current automatically satisfies the boundary condition and image vortices are unnecessary. However, in more recent numerical studies [32,33], imagelike vortices have been shown to be necessary for describing more accurately the velocity field near the boundaries. In the present work the rings of vortices are formed far enough from the boundaries so that the effects of their images can be neglected. We have numerically checked that this correction is negligible for all of the rings we have obtained. As an example for the 7-vortex ring we have mentioned above, we can overestimate the contribution of its image by locating it at a radius $r^{*}$ $=R^{2} / r_{0}[27,34]$ using $R=15$ which is smaller than the actual radius of the condensate. This correction to the velocity field roughly yields $4 \times 10^{-5} \hbar / m$ which is much smaller than $v$ $=0.41 \hbar / \mathrm{m}$. 


\section{VORTEX VELOCITY IN INHOMOGENEOUS MEDIA: ANALYTICAL EXPRESSIONS}

In this section we test the accuracy and applicability of the available formulas for the vortex velocity in inhomogeneous media. In a recent work [25], Sheehy and Radzihovsky have derived an approximate expression for the velocity field induced by a single vortex in an inhomogeneous twodimensional condensate. We shall refer to it as the background velocity $\mathbf{v}_{B}(\mathbf{r})$. This velocity does not include the divergent part due to the vortex itself. If $\mathbf{v}(\mathbf{r})$ denotes the total velocity field, then

$$
\mathbf{v}_{B}(\mathbf{r})=\mathbf{v}(\mathbf{r})-\frac{\hbar}{m} \frac{1}{\left|\mathbf{r}-\mathbf{r}_{0}\right|^{2}} \hat{\mathbf{z}} \times\left(\mathbf{r}-\mathbf{r}_{0}\right) .
$$

For the above-mentioned purpose, Sheehy and Radzihovsky define the variable $\rho_{s}$ as the smoothly varying superfluid density, which, in our case, may be well approximated by the ground-state density, as it can be seen from Figs. 3 and 4 . In terms of this density the background velocity field near the vortex core [25] is given by

$$
\mathbf{v}_{B}(\mathbf{r}) \sim \frac{\hbar}{2 m} \frac{\hat{\mathbf{z}} \times \boldsymbol{\nabla} \rho_{s}\left(\mathbf{r}_{0}\right)}{\rho_{s}\left(r_{0}\right)} \ln \left(\frac{\left|\mathbf{r}-\mathbf{r}_{0}\right|\left|\boldsymbol{\nabla} \rho_{s}\left(\mathbf{r}_{0}\right)\right|}{2 \rho_{s}\left(r_{0}\right)}\right),
$$

where $\mathbf{r}_{0}$ denotes the vortex position.

In the same work, the authors assumed that the vortex moves with this background velocity evaluated at $\left|\mathbf{r}-\mathbf{r}_{0}\right|$ $\sim l_{c}\left(r_{0}\right)$, where $l_{c}$ is the size of the core. We want to note that the field $\mathbf{v}_{B}$ given by expression (18) has been obtained under the assumption that $\boldsymbol{\nabla} \cdot \mathbf{j}=0, \mathbf{j}$ being the particle current density. Taking into account the continuity equation, this approximation is valid only outside the vortex core, where $\partial \rho_{v} / \partial t=0$. Thus, this velocity field should be evaluated at least at the border of the core but not inside it where $\partial \rho_{v} / \partial t \neq 0$.

If one assumes that this size $\left(l_{c}\right)$ is of the order of the healing length $\xi=\left[8 \pi \rho_{s}\left(r_{0}\right) a\right]^{-1 / 2}$, the following expression for the vortex precession velocity is obtained:

$$
\mathbf{v}_{p 1} \sim \frac{\hbar}{2 m} \frac{\hat{\mathbf{z}} \times \boldsymbol{\nabla} \rho_{s}\left(\mathbf{r}_{0}\right)}{\rho_{s}\left(r_{0}\right)} \ln \left(\frac{\xi\left|\boldsymbol{\nabla} \rho_{s}\left(\mathbf{r}_{0}\right)\right|}{2 \rho_{s}\left(r_{0}\right)}\right) .
$$

While, from our numerical calculations we have observed that this border is about $\left|\mathbf{r}-\mathbf{r}_{0}\right| \sim 5 \xi\left(r_{0}\right)$ where the form factor acquires the value $f \sim 0.95$, and thus this value seems to be a better estimate for describing the size of the core. Moreover, in a recent work [28], Nilsen et al. have found that in order to obtain a better approximation to the precession velocity to that of the background velocity, when the core size is not negligible, a new term should be added. Taking into account these considerations we alternatively propose the following expression:

$$
\mathbf{v}_{p 2} \sim \frac{\hbar}{2 m} \frac{\hat{\mathbf{z}} \times \boldsymbol{\nabla} \rho_{s}}{\rho_{s}} \ln \left(5 \frac{\xi\left|\nabla \rho_{s}\right|}{2 \rho_{s}}\right)-\frac{\hbar}{2 m} \frac{\hat{\mathbf{z}} \times \boldsymbol{\nabla} \rho_{s}}{\rho_{s}},
$$

where the second term of the above equation corresponds to the core contribution [28].

It may be seen that the last approximation is much better than the previous, especially when the core size is not neg-

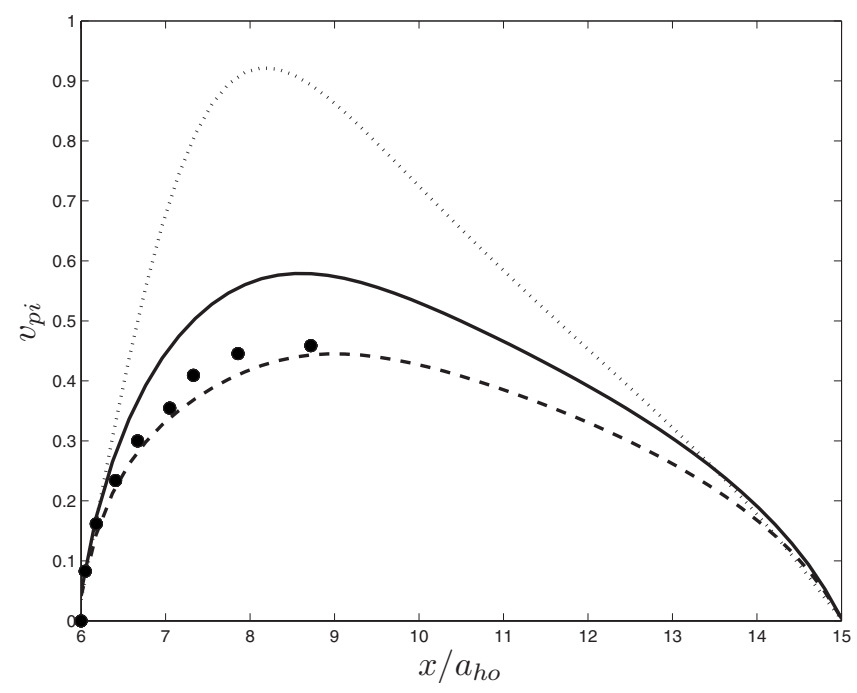

FIG. 6. Contributions to the vortex velocity, in units of $\hbar /\left(m a_{\text {ho }}\right)$, for a condensate with $N_{1}=2.5 \times 10^{5}$ atoms as a function of the stationary vortex position $x_{0}$. The dots indicate the velocity due to the presence of other vortices that arises from Eq. (15), while the solid, dashed, and dotted lines correspond to the analytical expressions $\mathbf{v}_{p 1}, \mathbf{v}_{p 2}$, and $\mathbf{v}_{p 3}$, respectively.

ligible, where the density of particles is lower. Other corrections to the precession velocity could also arise from imagelike vortices, as has been discussed for the $2 \mathrm{D}$ harmonic trapping potential in Ref. [33], but a general predictive formula that takes into account global effects is still not available.

Finally, a commonly used approximation [24,25,27] consists in replacing the argument inside the logarithm by a constant, namely, by the ratio between the size of the condensate and a typical healing length value. In particular, for example, for a condensate confined in a harmonic trapping potential this constant is $\left(R_{\mathrm{TF}} / \xi\right)$ being $R_{\mathrm{TF}}$ the TF radius and $\xi$ is evaluated at the center of the condensate. This yields

$$
\mathbf{v}_{p 3}=-\frac{\hbar}{2 m} \frac{\hat{\mathbf{z}} \times \boldsymbol{\nabla} \rho_{s}}{\rho_{s}} \ln \left(R_{\mathrm{TF}} / \xi\right) .
$$

In Fig. 6 (Fig. 7) we plot the vortex velocity field as a function of the position in a condensate with $N_{1}=2.5 \times 10^{5}$ $\left(N_{2}=10^{6}\right)$ atoms. Full circles correspond to the velocity given by Eq. (15) evaluated at the equilibrium position $x_{0}$ $=r_{0}$ of each $N_{v}$-vortex annular array configuration (see Table I). The modulus of the vortex precession velocities $\mathbf{v}_{p i}(i$ $=1,2,3)$ obtained from Eqs. (19)-(21), are also displayed. Note that the vectors $\mathbf{v}_{p i}$ evaluated on the $x$ axis between 6 $<x<15$ point towards negative values along the $y$ direction, and this is the only region in which the velocity given by the other vortices could be canceled.

From Figs. 6 and 7, it can be seen that the curve that better fits the velocity values is $\mathbf{v}_{p 2}$. It is important to remark that the three approximations of the velocity field work better when the number of particles is larger, and therefore the size of the core is smaller. In the present case the approximations are better for $N_{2}$ (see Fig. 7). 


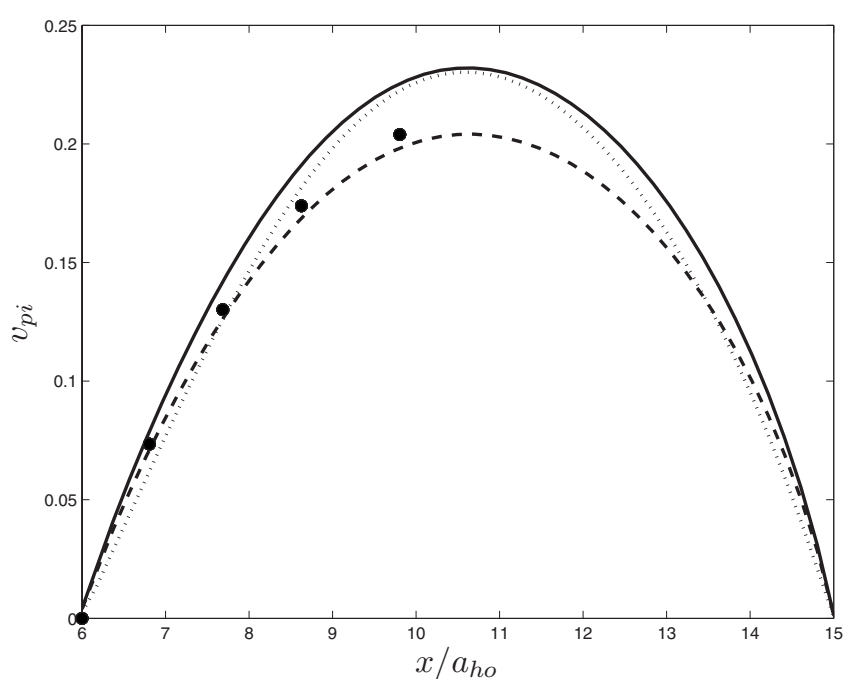

FIG. 7. Contributions to the vortex velocity, in units of $\hbar /\left(m a_{\mathrm{ho}}\right)$, for a condensate with $N_{2}=10^{6}$ atoms as a function of the stationary vortex position $x_{0}$. The same curves as in Fig. 6 are displayed.

Although these expressions were obtained for a 2D system, we have numerically found that they seem quite accurate to estimate the vortex precession velocity in the pancake-shaped condensate here considered. This suggests that in our case other corrections as, e.g., those induced by the image vortex, do not contribute much to the velocity field. However, these effects could play a more important role in other confined geometries [29] and/or for other system parameters and hence we believe that more efforts should be devoted to obtain more general predictive expressions which take into account also global effects, even for the $2 \mathrm{D}$ system.

\section{SUMMARY AND CONCLUDING REMARKS}

We have numerically obtained the possible stationary rings of vortices in a pancake-shaped BEC for two different number of particles and we have tested the accuracy of the available formulas for the vortex precession velocity derived for two-dimensional systems.

With respect to the type of arrays we have obtained we find that a larger vorticity is obtained with the lower number of particles. This can also be interpreted in terms of the velocity field due to the inhomogeneity. The expressions given by Eqs. (19)-(21) are proportional to the ratio between the density gradient and the density.

The value of the density in a given point of the space becomes smaller, the smaller the number of particles in the condensate. Whereas, reminding the reader that we are working in the TF regime, the value of the gradient of the density is minus the gradient of the potential and thus in that point remains the same independently of the number of trapped atoms. Therefore, a smaller number of particles provides a larger accessible inhomogeneity induced velocity to balance the velocity field due to the other vortices and therefore the number of vortices of the ring may be increased.

In connection to the vortex precession velocity. We have obtained a very simple formula for the velocity field generated by other vortices. This formula together with the fact that the vortex array is stationary, have enabled us to construct a very suitable scenario for exploring the accuracy of the available approximated expressions for the vortex precession velocity in trapped systems.

\section{ACKNOWLEDGMENTS}

This work has been performed under Grant No. PIP 5409 from CONICET, Grant No. X607 from UBA, Grant No. FIS2008-00421 from MEC (Spain), and Grant No. 2005SGR-00343 from Generalitat de Catalunya.
[1] R. J. Donnelly, Quantized Vortices in Helium II (Cambridge University Press, Cambridge, 1991).

[2] A. L. Fetter and A. A. Svidzinsky, J. Phys.: Condens. Matter 13, R135 (2001).

[3] C. J. Pethick and H. Smith, Bose-Einstein Condensation in Dilute Gases (Cambridge University Press, Cambridge, 2002).

[4] L. Pitaevskii and S. Stringari, Bose-Einstein Condensation (Clarendon, Oxford, 2003).

[5] A. L. Fetter, Laser Phys. 18, 1 (2008).

[6] M. R. Matthews, B. P. Anderson, P. C. Haljan, D. S. Hall, C. E. Wieman, and E. A. Cornell, Phys. Rev. Lett. 83, 2498 (1999).

[7] D. R. Scherer, C. N. Weiler, T. W. Neely, and B. P. Anderson, Phys. Rev. Lett. 98, 110402 (2007).

[8] C. Ryu, M. F. Andersen, P. Cladé, Vasant Natarajan, K. Helmerson, and W. D. Phillips, Phys. Rev. Lett. 99, 260401 (2007).

[9] B. P. Anderson, P. C. Haljan, C. A. Regal, D. L. Feder, L. A. Collins, C. W. Clark, and E. A. Cornell, Phys. Rev. Lett. 86, 2926 (2001)

[10] A. E. Leanhardt, A. Gorlitz, A. P. Chikkatur, D. Kielpinski, Y.
Shin, D. E. Pritchard, and W. Ketterle, Phys. Rev. Lett. 89, 190403 (2002)

[11] S. Tung, V. Schweikhard, and E. A. Cornell, Phys. Rev. Lett. 97, 240402 (2006).

[12] V. Bretin, S. Stock, Y. Seurin, and J. Dalibard, Phys. Rev. Lett. 92, 050403 (2004).

[13] S. Stock, B. Battelier, V. Bretin, Z. Hadzibabic, and J. Dalibard, Laser Phys. Lett. 2, 275 (2005).

[14] M. Cozzini, B. Jackson, and S. Stringari, Phys. Rev. A 73, 013603 (2006).

[15] A. Aftalion and I. Danaila, Phys. Rev. A 69, 033608 (2004).

[16] A. L. Fetter, B. Jackson, and S. Stringari, Phys. Rev. A 71, 013605 (2005).

[17] J. K. Kim and A. L. Fetter, Phys. Rev. A 72, 023619 (2005).

[18] H. Fu and E. Zaremba, Phys. Rev. A 73, 013614 (2006).

[19] M. Möttönen, S. M. M. Virtanen, T. Isoshima, and M. M. Salomaa, Phys. Rev. A 71, 033626 (2005).

[20] V. Pietilä, M. Möttönen, T. Isoshima, J. A. M. Huhtamäki, and S. M. M. Virtanen, Phys. Rev. A 74, 023603 (2006). 
[21] L. C. Crasovan, V. Vekslerchik, V. M. Pérez-García, J. P. Torres, D. Mihalache, and L. Torner, Phys. Rev. A 68, 063609 (2003).

[22] L. C. Crasovan, V. M. Pérez-García, I. Danaila, D. Mihalache, and L. Torner Phys. Rev. A 70, 033605 (2004).

[23] D. M. Jezek, P. Capuzzi, and H. M. Cataldo, J. Phys. B 41, 045304 (2008).

[24] A. A. Svidzinsky and A. L. Fetter, Phys. Rev. A 62, 063617 (2000).

[25] D. E. Sheehy and L. Radzihovsky, Phys. Rev. A 70, 063620 (2004).

[26] D. E. Sheehy and L. Radzihovsky, Phys. Rev. A 70, 051602(R) (2004).

[27] E. Lundh and P. Ao, Phys. Rev. A 61, 063612 (2000).
[28] H. M. Nilsen, G. Baym, and C. J. Pethick, Proc. Natl. Acad. Sci. U.S.A. 103, 7978 (2006).

[29] P. Mason, N. G. Berloff, and A. L. Fetter, Phys. Rev. A 74, 043611 (2006).

[30] W. H. Press, S. A. Teukolsky, W. T. Vetterling, and B. P. Flannery, Numerical Recipes in Fortran 77: The Art of Scientific Computing (Cambridge University Press, Cambridge, 1992).

[31] J. K. Kim and A. L. Fetter, Phys. Rev. A 70, 043624 (2004).

[32] P. Mason and N. G. Berloff, Phys. Rev. A 77, 032107 (2008).

[33] D. M. Jezek and H. M. Cataldo, Phys. Rev. A 77, 043602 (2008).

[34] M. Guilleumas and R. Graham, Phys. Rev. A 64, 033607 (2001). 